\title{
LAS AVES DE LOS HUMEDALES DE ETEN, LAMBAYEQUE, PERÚ
}

\section{BIRDS OF ETEN MARSHES, LAMBAYEQUE, PERÚ}

\author{
Fernando Angulo-Pratolongo ${ }^{1}$, Thomas S. Schulenberg ${ }^{2}$ y Elberth Edevaly Puse-Fernández ${ }^{3}$
}

\begin{abstract}
Resumen
Se presenta la avifauna de los humedales de Eten, Lambayeque, Perú, los cuales representan el límite norte de los humedales dominados por Totora (Typha angustifolia) y Junco (Scirpus spp.) en la costa peruana. Para el presente trabajo se han definido tres tipos de hábitats: Humedal; Mar y playa; y Arbustos, árboles y agricultura. La lista de aves recoge información de observaciones entre los años 1978 y 2009 y está compuesta por 153 especies pertenecientes a 17 órdenes y 45 familias. Las familias con mayor representatividad de especies son Scolopacidae con 21 especies y Laridae con 15. Cuatro especies son endémicas tumbesinas y una es endémica tumbesina y del Perú. Una especie está amenazada globalmente y ocho a nivel nacional. Constituyen amenazas para el área la cacería no regulada, la alta incidencia de residuos sólidos, la ganadería y el avance de la agricultura. Se propone que los humedales de Eten sean un Área de Importancia para la Conservación de Aves (IBA) y reciban protección formal a través del gobierno regional de Lambayeque. Futuras investigaciones en el área deben apuntar a establecer un adecuado manejo del área en cuanto a extracción de recursos y uso turístico, así como determinar si es que Sternula lorata, especie globalmente amenazada, anida en este lugar.
\end{abstract}

Palabras clave: Eten, Lambayeque, avifauna, humedales costeros, Sternula lorata, conservación.

\begin{abstract}
We present a list of the birds of the Eten marshes, Lambayeque, Perú. These marshes are the northernmost limit of marshes dominated by Typha angustifolia and Scirpus spp. on the Peruvian coast. Three habitat types exist on the study area: marshes; sea and beach; and trees, shrubs and agriculture. The bird list gathers information from observations made between 1978 and 2009 and is composed by 153 species belonging to 17 orders and 45 families. Families best represented were Scolopacidae with 21 species and Laridae with 15. Five species are Tumbesian endemics, one of which is also endemic to Perú. One species is globally threatened and eight are threatened according to national legislation. The problems of the area are unregulated hunting, a high amount of solid residues, cattle ranching and expansion of agriculture. We propose the Eten marshes as an Important Bird Area and discuss that it should receive formal protection through the Lambayeque regional government. Future research must be directed to establish a sustainable management of the area for resource extraction and for tourist use. Also, it is important to determine if Sternula lorata, a globally threatened species nests in this area.
\end{abstract}

Key words: Eten, Lambayeque, Sternula lorata.

\section{Introducción.}

El presente trabajo tiene como objetivo dar a conocer la avifauna de los humedales de Eten, Lambayeque, Perú. Esta fue compilada como parte del expediente técnico de categorización de dichos humedales para ser designada "Área de Conservación Regional” por el Gobierno Regional de Lambayeque (GRL). El área fue originalmente declarada como “Área Ecológica de Interés Regional Humedales de Eten”, a través de la gerencia de Recursos Naturales y Gestión del Medio Ambiente del GRL, por la ordenanza $\mathrm{N}^{\circ}$ 004-2005 (sobre 1377 ha) en los distritos de Monsefú, Puerto Eten y Ciudad Eten. El propósito de dicha acción fue conservar el ecosistema de humedales, incluida la flora y fauna, así como las manifestaciones arqueológicas de esta zona.
Los humedales de Eten son, dentro de la costa peruana, el límite norte de los humedales dominados por Totora (Typha angustifolia L.) y Junco (Scirpus spp. L.). Siguiendo la línea de costa desde Eten hacia el norte, los siguientes humedales costeros son el Estuario de Virrilá, los manglares de San Pedro de Vice, y los manglares de la desembocadura de los ríos Tumbes y Zarumilla. En ninguno de estos sitios se presentan las características de vegetación presentes en los humedales de Eten (Morrison \& Ross, 1989).

Ornitológicamente, los humedales de Eten fueron explorados en 1899 (Chubb, 1919) y durante 1978 y 1979 (Schulenberg \& Parker, 1981). Durante el censo de aves playeras neárticas en la costa peruana hecho en 1986 por Morrison \& Ross (1989), éstos mencionan que el área de Eten incluyó 453 de 455 individuos del género Tringa de la eco-unidad 
"Chiclayo" (comprendida entre Trujillo y la frontera con Ecuador), lo que representaba a su vez el 19.1\% del total de la costa pacífica. El tramo 65 (donde se encuentran Eten), está descrito por Morrison \& Ross (1989) como "Tierra adentro; laguna con áreas húmedas y humedal/ desierto arenoso". En este tramo se contaron 1334 aves playeras, de las cuales 800 fueron Calidris alba (Pallas, 1764). Se cita además, que grandes números de gaviotas fueron registradas en las lagunas de estos humedales. Pulido et al. (1996) señalan que la boca del río Eten (río Reque, humedales de Eten) es un humedal de importancia nacional de la costa peruana y es de reconocida importancia para las aves migratorias, por lo que se le debe conservar utilizando los instrumentos legales disponibles en el Perú.

En este trabajo se presenta la lista de aves de los humedales de Eten, se comenta sobre las especies de interés, se hace un breve análisis del estado de conservación del humedal y se presentan recomendaciones para su futura conservación.

\section{Materiales y métodos.}

Área de estudio.

Los humedales de Eten se encuentran políticamente entre los distritos de Monsefú, Puerto Eten, Ciudad Eten y Santa Rosa, en la provincia de Chiclayo, departamento de Lambayeque. El área de estudio se encuentra a $17 \mathrm{~km}$ en dirección suroeste de la ciudad de Chiclayo y abarca una extensión de aproximadamente 1400 hectáreas comprendidas entre los 6 $6^{\circ} 53^{\prime} 40^{\prime}$ ' LS/ 79 53' 41” LO (extremo Norte) y los 6 $6^{\circ} 55^{\prime} 19^{\prime}$ LS/ $79^{\circ}$ 52' 22” LO (extremo Sur) y entre 0 y 15 msnm (Figura 1). Esto incluye a los humedales propiamente dichos, la franja arenosa colindante con el mar, y las áreas agrícolas y arbustivas semi desérticas que rodean el humedal.

Los humedales de Eten se forman por el afloramiento de aguas del río Reque (conocido como Chancay en las partes altas de la cuenca) alrededor de la desembocadura del mismo en el Océano Pacifico. Se ubican dentro de la ecorregión "Bosques secos de Piura y Tumbes", muy cerca del límite con la ecorregión "Desierto de Sechura" (CDC-UNALM, 2006). Dentro del área de estudio, se han definido para el presente artículo los siguientes tipos de hábitat:

Humedal $(\mathrm{H})$ : Lagunas de agua dulce o salobre y áreas de suelo húmedo cubiertas principalmente con vegetación de las especies Totora (Typha angustifolia) y Junco (Scirpus spp.). Además, se incluye en esta categoría a los drenes y a las áreas cubiertas de vegetación sobre la arena, que incluye Lejía (Batis marítima L.), Platanito (Sessuvium portulacastrum (L) L.), Grama Salada (Distichlis spicata L. Greene), Verdolaga (Salicornia fruticosa (L) L.) y las gramíneas Paspalum vaginatum Sw. y Sporobolus virginicus (L) Kunth.

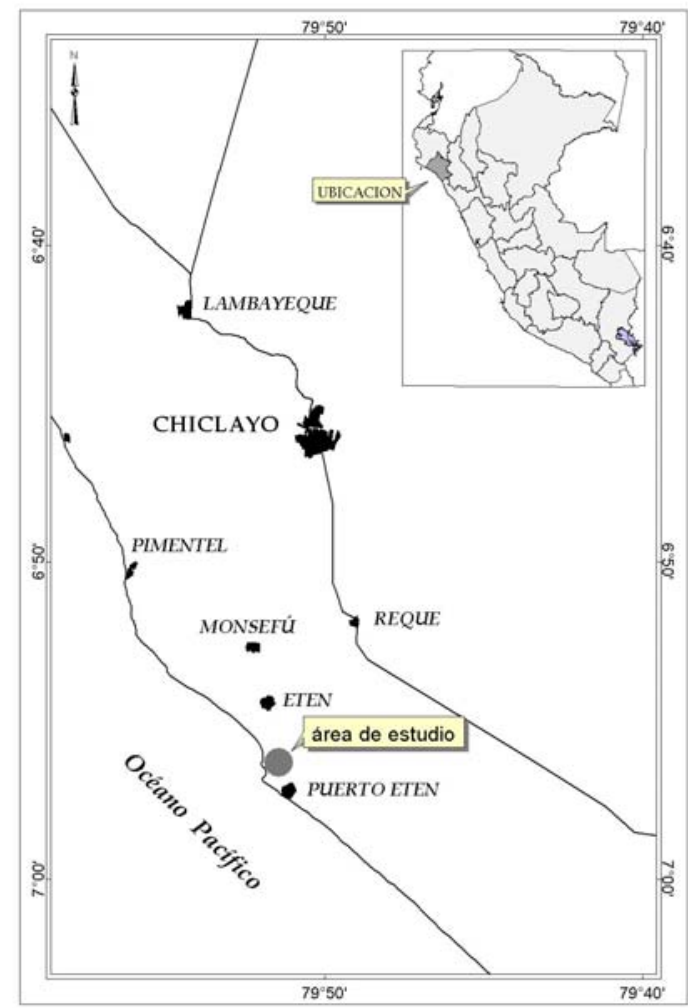

Figura 1. Mapa de ubicación de los humedales de Eten.

Mar y playa (M): Incluye al Océano Pacifico, que se encuentra al oeste de los humedales y la franja de playa de arena (de $100 \mathrm{~m}$ de ancho en promedio), hasta el límite en que empieza el humedal y las lagunas. En este hábitat no hay vegetación.

Arbustos, árboles y agricultura (A): Incluye las áreas con cultivos agrícolas y las áreas cubiertas de vegetación arbórea y arbustiva que se encuentran rodeando a los humedales hacia el este. Los cultivos principales son maíz (Zea mays L.), camote (Ipomoea batatas (L.) Lam.), alfalfa (Medicago sativa L.), chileno (Dolichos lablab L.), caña de azúcar (Saccharum officinarum L.) y sandía (Citrullus lanatus (Thunb.) Matsum. et Nakai) y las áreas cubiertas de vegetación arbórea y arbustiva (en parches o sirviendo como cerco entre las áreas agrícolas) están compuestas principalmente por las especies chilco (Baccharis spp.), chope (Cryptocarpus pyriformis Kunth), algarrobo (Prosopis pallida Benth.) y guaba (Inga edulis Mart.).

Metodología.

Se ha compilado la lista de aves de los humedales de Eten en base a tres fuentes de información: observaciones hechas por los autores, registros proporcionados por otros observadores y revisión de la bibliografía. Las observaciones de los autores incluyen los años 2004-09 (Fernando Angulo Pratolongo (FAP)), 1978-79, 1985-86, 1994 у 2005 
(Thomas S. Schulenberg (TSS)), y 2006-2008 (Elberth Edevaly Puse Fernández (EEPF)). Registros de otros observadores incluyen los años 1997 - 2008 y la bibliografía consultada corresponde a Chubb (1919) y Schulenberg \& Parker (1981). Las diferentes fuentes de registro en conjunto, cubren todos los meses del año.

En la lista de aves se señala en qué tipo de hábitat se ha registrado cada especie, según la descripción y abreviaturas señaladas en la sección anterior. Asimismo, se hace referencia a las especies que son endémicas de la región de endemismo Tumbesina (Endemic Bird Area, EBA por sus siglas en inglés) (Stattersfield et al., 1998) y/o del Perú (Schulenberg et al., 2007). También se señalan las especies que están amenazadas globalmente (BirdLife International, 2010) o a nivel nacional (El Peruano, 2004).

\section{Resultados y discusión.}

La lista de las aves de los humedales de Eten se presenta en la Tabla 1. Esta sigue la nomenclatura vigente del South American Classification Comité (SACC) (Remsen et al., 2009). Los nombres en castellano son tomados de Plenge (2009). Adicionalmente, cuando existen, se señalan los nombres locales de las especies.

Se ha registrado un total de 153 especies, las cuales pertenecen a 17 órdenes y 45 familias. Las familias con mayor representatividad de especies fueron Scolopacidae (playeros) con 21 especies (14 \%) y Laridae (gaviotas y gaviotines) con 15 especies (10 \%). Juntas ambas familias representan el $24 \%$ de la avifauna de Eten. En la Figura 2 se muestran las familias con mayor número de representantes.

Con respecto al endemismo, cuatro son endémicas de la EBA Tumbesina: Forpus coelestis (Lesson, 1847), Myrmia micrura (Gould, 1854), Myiodynastes bairdii (Gambel, 1847) y Thryothorus superciliaris
(Lawrence, 1869), mientras que una especie es endémica tanto de la EBA Tumbesina como del Perú: Geositta peruviana (Lafresnaye, 1847). Con respecto al estado de amenaza, según la clasificación global (BirdLife, 2010), una especie está clasificada como amenazada: Sternula lorata (Philippi \& Landbeck, 1861), clasificada como "En Peligro", mientras que según la legislación peruana, cuatro especies están clasificadas como "En Peligro": Pelecanus thagus (Molina, 1782), Sula variegata (Tschudi, 1843), Phalacrocorax gaimardi (Lesson \& Garnot, 1828), Phalacrocorax bougainvillii (Lesson, 1837) y Platalea ajaja (Linnaeus, 1758) y cuatro como "Vulnerable": Netta erythrophthalma (Wied, 1832), Sternula lorata, Larosterna inca (Lesson, 1827) y Sterna hirundinacea (Lesson, 1831).

En lo referente al uso del hábitat (Tabla 1), se ha encontrado que 29 especies usan exclusivamente el Humedal; 42 la vegetación de Arbustos, árboles y agricultura y 12 el Mar y playa (Figura 3). El número de especies que usan el hábitat Humedal (total o parcialmente) totaliza las 99, lo cual representa el 65\% de las especies que ocurren en los humedales de Eten. Dos especies usan los tres hábitats: Falco peregrinus (Tunstall, 1771) e Himantopus mexicanus (Müller, 1776).

Especies de interés.

Netta erythrophthalma. Esta especie está disminuyendo en Sudamérica, pero debido a que también ocurre en África, se encuentra a nivel global en la categoría de "Preocupación menor" (BirdLife International, 2010). A nivel nacional, está en la categoría de "Vulnerable" (El Peruano, 2004). En la actualidad se encuentra extinta de la costa del Perú y existen escasos reportes actuales de la especie en todo el Perú, excepto por registros sin confirmar en varias lagunas de Puno y Cuzco (Velarde, 1998). Poco se sabe acerca de su antigua distribución en Perú, siendo la mayor parte de los registros provenientes de humedales costeros de Lambayeque, Lima y Arequipa. Sin embargo, no ha sido confiablemente reportada de la costa del Perú desde 1962 (Hughes, 1970). En Eten, Chubb (1919) menciona que un macho adulto de Netta erythrophthalma (listado como Nyroca nationi en dicho artículo) fue colectada el 11 de octubre de 1899. A pesar de que no ha sido reportada en Lambayeque desde 1935, su anterior presencia en Eten demuestra la importancia de este sitio para las aves acuáticas en el Perú. Durante el periodo en que se realizaron las observaciones citadas en este trabajo, esta 
especie no fue registrada.

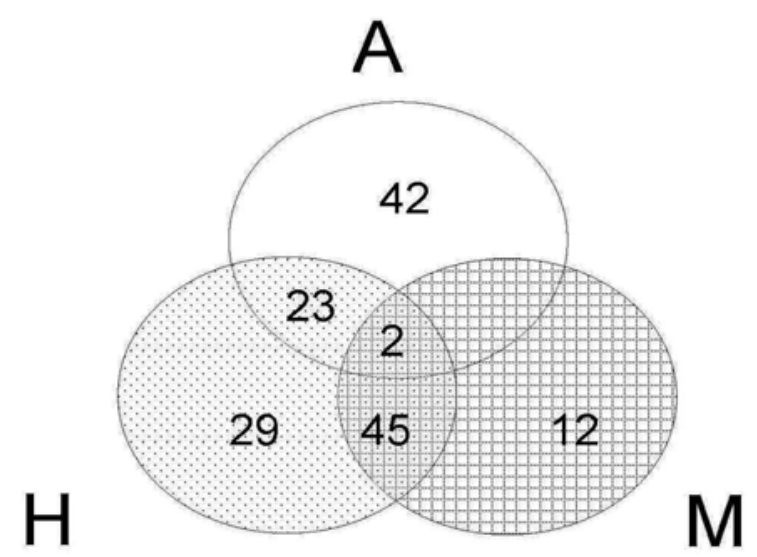

Figura 3. Uso del hábitat por las aves de los humedales de Eten.

Hábitat. H: Humedal, M: Mar y playa, A: Arbustos, árboles y agricultura.

Himantopus mexicanus. EEPF observó y filmó en febrero del 2007 dos individuos de la subespecie melanurus, la cual se diferencia de $H$. m. mexicanus por tener la corona y una franja en la espalda de color blanco. La subespecie regularmente registrada en Eten es H. m. mexicanus. Schulenberg et al. (2007) reporta melanurus como reproductor en la costa sur del Perú, llegando por el norte hasta Lima. Es posible que los individuos de la subespecie melanurus reportados en Eten sean vagabundos que han viajado por la costa hacia el norte o que hayan llegado del lado este de los Andes cruzando la cordillera.

Chroicocephalus cirrocephalus (Vieillot, 1818). Esta especie fue registrada reproduciéndose en Eten por primera vez a principios de agosto de 1994, cuando TSS encontró 20 juveniles, algunos aparentemente incapaces de volar y un pichón. EEPF encontró nidos con huevos en febrero de 2007 y pichones en marzo y abril del mismo año. Esta especie no fue reportada como reproductora en el Perú hasta 1970 (Tovar \& Ashmole, 1970) en Laguna Chica, Ica, siendo Eten uno de los sitios confirmados.

Sternula lorata. Esta especie se encuentra en la categoría de "En Peligro" (BirdLife International, 2010) a nivel global y como "Vulnerable" (El Peruano, 2004) a nivel nacional. La presencia de esta especie en los humedales de Eten fue reportada por Chubb (1919) (listado como Sterna lorata en dicho artículo), quien menciona que once machos y una hembra, adultos e inmaduros, fueron colectados en Septiembre de 1899.

Durante el periodo en que se realizaron las observaciones de este trabajo, esta especie ha sido reportada 8 veces entre octubre de 1978 y noviembre de 2007 (Tabla 2). En septiembre de 2006 fueron observados 2 pichones emplumados además de 5 individuos adultos (R. Webster \& R. Rowlett com. pers.). El estimado poblacional para la especie en Eten es de 30-40 individuos. Si bien, a la fecha no se ha reportado este sitio como lugar de anidamiento para la especie (Zavalaga et al., 2009), es posible que hayan colonias en las cercanías de Eten.

Discusión.

Los humedales de Eten contienen una comunidad de aves típica de humedales costeros, con una fuerte influencia de especies que viven en el hábitat de Arbustos, árboles y agricultura, debido a que este humedal se encuentra rodeado parcialmente de este tipo de hábitat, a diferencia de otros humedales costeros que están rodeados de áreas semi desérticas y/o arenosas.

Durante las salidas de campo se ha observado que la problemática de estos humedales es la presencia de cacería no regulada, la cual se da con armas de fuego principalmente sobre especies de la familia Anatidae y también sobre Phoenicopterus chilensis (Molina, 1782). También existe cacería con perros, mayormente sobre juveniles de Gallinula chloropus (Linnaeus, 1758) (Rallidae).

Otro problema de Eten es la alta incidencia de residuos sólidos y de desmonte. Es necesario prohibir (a nivel municipal y regional) usar este lugar como botadero y realizar campañas de limpieza. Asimismo, la ganadería y el avance de la agricultura son factores importantes que afectan la conservación de estos humedales. Estas actividades, que ocurren en creciente aumento, vienen deteriorando la calidad y reduciendo el área del humedal de una forma dramática.

Por otro lado, en Eten se lleva a cabo la extracción de Junco (Scirpus spp.) y Totora (Typha angustifolia) por parte de los pobladores locales para su uso en la elaboración de artesanías y utensilios. Esta actividad debe permitirse bajo un plan de manejo y no de otra forma, en zonas específicas y en cantidades reguladas, para así evitar el impacto negativo en las poblaciones de las aves, ya que ha sido registrada en los lugares de anidamiento de éstas.

Los humedales de Eten son un área que viene siendo usada para la observación de aves (aviturismo o birdwatching) por los tours que realizan la ruta Norte del Perú. Esta actividad puede ser una

Tabla 2. Registros de Sternula lorata en los Humedales de Puerto Eten, Lambayeque, Perú.

\begin{tabular}{ccl}
\hline Fecha & $\mathrm{N}^{\mathrm{o}}$ Ind. & Fuente \\
\hline Oct-78 & 12 & TSS \\
Nov-78 & 3 & TSS \\
Jul-79 & 40 & TSS \\
Sep-97 & 25 & R. Webster \& R. Rowlett \\
Sep-02 & 1 & R. Webster \& R. Rowlett \\
Sep-06 & 7 & R. Webster \& R. Rowlett \\
Oct-06 & 15 & FAP (Zavalaga et al., 2009) \\
Nov-07 & 2 & R. Webster \& R. Rowlett \\
\hline
\end{tabular}


importante fuente de ingresos para los pobladores locales (guías y cobro de entradas). Para ello se necesita trabajar en entrenar a pobladores locales en la observación de aves, mejorar las condiciones de seguridad, determinar la ubicación de miradores, demarcar circuitos y caminos y asegurar la conservación del área.

El programa de las Áreas Importantes para la Conservación de las Aves (IBAs) es una iniciativa a nivel mundial que se centra en la identificación, documentación y conservación de una red global de sitios críticos para la conservación de las aves y biodiversidad (Devenish et al., 2009). Los humedales de Eten cumplen el criterio A1 para ser considerados como un Área de Importancia para la Conservación de Aves (IBA por las siglas en inglés). Este criterio tiene por definición: "El sitio mantiene regularmente una población significativa de una o más especies amenazadas a nivel mundial u otras especies cuya conservación es de interés global. La presencia regular de una especie "En Peligro Critico" o "En Peligro", independientemente del tamaño de su población en un sitio, es suficiente para proponer el sitio como IBA" (BirdLife International \& Conservation International, 2005). Para el caso de Eten, existe una población residente de Sternula lorata, la cual tiene al menos 3040 individuos. Por esta razón, se propone que este lugar sea considerado una IBA. El Gobierno Regional de Lambayeque debe tomar la iniciativa y considerar asimismo, la propuesta de crear un área de conservación regional.

Futuras investigaciones en los humedales de Eten deberían apuntar a determinar las cifras de las poblaciones de aves, la reproducción y estacionalidad de éstas y definir las zonas de anidamiento, para poder establecer un adecuado manejo del área en cuanto a extracción de recursos y uso turístico. Asimismo, considerando que Sternula lorata ha sido recientemente reportada anidando en humedales costeros (Laguna El Paraíso) (Zavalaga et al., 2009), se debe investigar si esta especie anida en los humedales de Eten o en las cercanías, ya que pichones emplumados han sido observados. De confirmarse el anidamiento de esta especie en Eten, aumentaría la importancia de este lugar.

\section{Agradecimientos.}

Se agradece al personal de la gerencia de Recursos Naturales y Gestión del Medio Ambiente del Gobierno Regional de Lambayeque por las facilidades prestadas para visitar el área. Agradecemos también a J. Barrio, A. Dattner, A. Huamán, M. Reque, R. A. Rowlett, E. Tejada, P. Venegas, R. Webster y P. Yrigoyen por compartir sus observaciones hechas en los humedales de Eten y su compañía en el campo. Asimismo, agradecemos a dos revisores anónimos que ayudaron a mejorar el manuscrito.

\section{Literatura citada.}

BirdLife International \& Conservation International. 2005. Áreas Importantes para la Conservación de las Aves en los Andes Tropicales: sitios prioritarios para la conservación de la biodiversidad. Quito, Ecuador: BirdLife International (Serie de Conservación de BirdLife $\left.\mathrm{N}^{\circ} 14\right)$.

BirdLife International. 2010. 2010 IUCN Red List for birds. http://www.birdlife.org/datazone/species/

Centro de Datos para la Conservación - Universidad Nacional Agraria La Molina (CDC-UNALM). 2006. Análisis de la Cobertura Ecológica del Sistema Nacional de Áreas Naturales Protegidas por el Estado. Lima, Perú: CDC-UNALM/TNC.

Chubb C. 1919. Notes on collections of birds in the British Museum, from Ecuador, Peru, Bolivia and Argentina. Ibis 61:1-55.

Devenish C., Diaz Fernandez D.F., Clay R.P., Davidson I. \& I. Yepez Zabala. 2009. Important Bird Areas Americas Priority sites for biodiversity conservation. Quito, Ecuador: BirdLife International (BirdLife Conservation Series $\mathrm{N}^{\mathrm{o}} 16$ ).

El Peruano. 2004. Decreto Supremo No 034 - 2004 - AG del 22 de Septiembre de 2004. Categorización de Especies Amenazadas de Fauna Silvestre.

Hughes R.A. 1970. Notes on the birds of the Mollendo district, southwest Peru. Ibis 112: 239.

Morrison R.I.G. \& R. K. Ross. 1989. Atlas of Nearctic Shorebirds on the Coast of South America. Vols. 1 and 2, Canadian Wildlife Service Special Publication, Ottawa.

Plenge M.A. 2009. List of the birds of Peru. Lima, Perú.

Pulido V., Jahncke J., Makamatsu P. \& C. Flores. 1996. Conservation of Charadriiformes on the Peruvian coast. International Wader Studies 8:55-61.

Remsen J.V. Jr., Cadena C.D., Jaramillo A., Nores M., Pacheco J.F., Robbins M.B., Schulenberg T.S., Stiles F.G., Stotz D.F., \& Zimmer K.J. 2009. Version [24 June 2009]. A classification of the bird species of South America. American Ornithologists' Union.

http://www.museum.lsu.edu/ Remsen/SACCBaseline.html

Schulenberg T.S. \& Parker T.A. III. 1981. Status and distribution of some northwest Peruvian birds. Condor, 83: 209-216.

Schulenberg T., Stotz D., Lane D., O’Neill J. \& Parker T. 2007. Birds of Peru. Princeton University Press. New Jersey.

Stattersfield A.J., Crosby M.J., Long A.J. \& Wege D. 1998. Endemic birds areas of the world. Priorities for biodiversity conservation. BirdLife International. Cambridge, U.K. Conservation Series 7.

Tovar S.H. \& Ashmole N.P. 1970. A breeding record for the Gray-hooded Gull, Larus cirrocephalus, on the Peruvian coast. Condor, 72: 119-122.

Velarde D. 1998. Resultados de los Censos Neotropicales de Aves Acuáticas en el Perú 1992 - 1995. Programa de Conservación y Desarrollo Sostenido de Humedales, Perú. Lima, Perú.

Zavalaga C., Hardesty J., Mori G., Chávez-Villavicencio C. \& Tello A. 2009. Current status of Peruvian Terns Sternula lorata in Perú: threats, conservation and research priorities. Bird Conservation International 19: 175-186 
Tabla 1. Aves de los Humedales de Puerto Eten, Lambayeque, Perú.

Hábitat. H: Humedal, M: Mar y playa, A: Arbustos, árboles y agricultura Endemismo. T: EBA Tumbesina, P: Perú Amenaza. EN: En Peligro, VU: Vulnerable, LC: Preocupación menor

Familia Nombre Científico
Nombre en Español

Nombre en Ingles

\begin{tabular}{ll}
\hline ANATIDAE & Sarkidiornis melanotos (Pennant, 1769) \\
ANATIDAE & Anas georgica (Gmelin, 1789) \\
ANATIDAE & Anas bahamensis (Linnaeus, 1758) \\
ANATIDAE & Anas discors (Linnaeus, 1766) \\
ANATIDAE & Anas cyanoptera (Vieillot, 1816) \\
ANATIDAE & Netta erythrophthalma (Wied, 1832) \\
ANATIDAE & Oxyura jamaicensis ferruginea (Eyton, 1838) \\
PODICIPEDIDAE & Rollandia rolland (Quoy \& Gaimard, 1824) \\
PODICIPEDIDAE & Podilymbus podiceps (Linnaeus, 1758) \\
PODICIPEDIDAE & Podiceps major (Boddaert, 1783) \\
PODICIPEDIDAE & Podiceps occipitalis (Garnot, 1826) \\
PHOENICOPTERIDAE & Phoenicopterus chilensis (Molina, 1782) \\
PELECANIDAE & Pelecanus thagus (Molina, 1782) \\
PELECANIDAE & Pelecanus occidentalis (Linnaeus, 1766) \\
SULIDAE & Sula nebouxii (Milne-Edwards, 1882) \\
SULIDAE & Sula variegata (Tschudi, 1843) \\
PHALACROCORACIDAE & Phalacrocorax brasilianus (Gmelin, 1789) \\
PHALACROCORACIDAE & Phalacrocorax gaimardi (Lesson \& Garnot, 1828) \\
PHALACROCORACIDAE & Phalacrocorax bougainvillii (Lesson, 1837) \\
FREGATIDAE & Fregata magnificens (Mathews, 1914) \\
ARDEIDAE & Ixobrychus exilis (Gmelin, 1789) \\
ARDEIDAE & Nycticorax nycticorax (Linnaeus, 1758) \\
ARDEIDAE & Butorides striata (Linnaeus, 1758) \\
ARDEIDAE & Bubulcus ibis (Linnaeus, 1758) \\
ARDEIDAE & Ardea cocoi (Linnaeus, 1766) \\
ARDEIDAE & Ardea alba (Linnaeus, 1758) \\
ARDEIDAE & Egretta tricolor (Müller, 1776) \\
&
\end{tabular}

Pato Crestudo (Pato Real)

Pato Jergón

Pato Gargantillo (Pato Alabanco)

Pato de Ala Azul

Pato Colorado (Pato Lefe)

Pato Morado

Pato Andino

Zambullidor Pimpollo

Zambullidor de Pico Grueso

Zambullidor Grande

Zambullidor Plateado

Flamenco Chileno

Pelícano Peruano

Pelicano Marrón

Piquero de Patas Azules

Piquero Peruano

Cormorán Neotropical (Pato Cuervo)

Cormorán de Patas Rojas

Cormorán Guanay

Avefragata Magnífica

Mirasol Leonado

Huaco Común

Garcita Estriada (Tamanquita)

Garcita Bueyera

Garza Cuca

Garza Grande

Garcita Tricolor

\section{Comb Duck}

Yellow-billed Pintail

White-cheeked Pintail

Blue-winged Teal

Cinnamon Teal

Southern Pochard

Andean Duck

White-tufted Grebe

Pied-billed Grebe

Great Grebe

Silvery Grebe

Chilean Flamingo

Peruvian Pelican

Brown Pelican

Blue-footed Booby

Peruvian Booby

Neotropic Cormorant

Red-legged Cormorant

Guanay Cormoran

Magnificent Frigatebird

Least Bittern

Black-crowned Night-Heron

Striated Heron

Cattle Egret

Cocoi Heron

Great Egret

Tricolored Heron

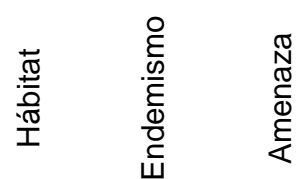

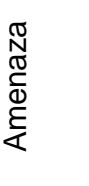

H

H

H

H

H

H

H

$H, M$

$H$

H, M

H, M

$M$

M

M

$\mathrm{H}, \mathrm{M}$

M

M

$M$

$\mathbf{H}$
$\mathbf{H}$

H

H

H

H, M

H
LC I VU

NT I EN

LC I EN

NT / EN

NT / EN 
Tabla 1. Aves de los Humedales de Puerto Eten, Lambayeque, Perú.

Hábitat. H: Humedal, M: Mar y playa, A: Arbustos, árboles y agricultura Amenaza. EN: En Peligro, vU: Vulnerable, LC: Preocupación menor

\begin{tabular}{|c|c|c|c|c|c|c|}
\hline Familia & Nombre Científico & Nombre en Español & Nombre en Ingles & $\begin{array}{l}\frac{\vec{\sigma}}{\stackrel{0}{0}} \\
\frac{\pi}{\pi} \\
\frac{\pi}{I}\end{array}$ & 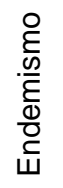 & 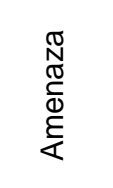 \\
\hline ARDEIDAE & Egretta thula (Molina, 1782) & Garcita Blanca & Snowy Egret & $\mathrm{H}, \mathrm{M}$ & & \\
\hline ARDEIDAE & Egretta caerulea (Linnaeus, 1758) & Garcita Azul & Little Blue Heron & H & & \\
\hline THRESKIORNITHIDAE & Plegadis ridgwayi (Allen, 1876) & Ibis de la Puna (Yanavico) & Puna Ibis & $\mathbf{H}$ & & \\
\hline THRESKIORNITHIDAE & Platalea ajaja (Linnaeus, 1758) & Espátula Rosada & Roseate Spoonbill & $\mathrm{H}, \mathbf{M}$ & & LC /EN \\
\hline CATHARTIDAE & Cathartes aura (Linnaeus, 1758) & Gallinazo de Cabeza Roja & Turkey Vulture & $\mathrm{H}, \mathrm{M}$ & & \\
\hline CATHARTIDAE & Coragyps atratus (Bechstein, 1783) & Gallinazo de Cabeza Negra & Black Vulture & $H, A$ & & \\
\hline PANDIONIDAE & Pandion haliaetus (Linnaeus, 1758) & Águila Pescadora & Osprey & $\mathrm{H}, \mathrm{M}$ & & \\
\hline ACCIPITRIDAE & Circus cinereus (Vieillot, 1816) & Gavilán Cenizo & Cinereous Harrier & $\mathbf{H}$ & & \\
\hline ACCIPITRIDAE & Buteogallus meridionalis (Latham, 1790) & Gavilán Sabanero (Gavilán Sonso) & Savanna Hawk & H, A & & \\
\hline ACCIPITRIDAE & Buteo polyosoma (Quoy \& Gaimard, 1824) & Aguilucho de Dorso Rojo & Red-backed Hawk & $\mathrm{H}, \mathrm{A}$ & & \\
\hline FALCONIDAE & Caracara cheriway (Jacquin, 1784) & Caracara Crestado (Guaraguau) & Crested Caracara & A & & \\
\hline FALCONIDAE & Falco sparverius (Linnaeus, 1758) & Cernícalo Americano & American Kestrel & $\mathbf{H}, \mathbf{A}$ & & \\
\hline FALCONIDAE & Falco peregrinus (Tunstall, 1771) & Halcón Peregrino & Peregrine Falcon & $H, A, M$ & & \\
\hline RALLIDAE & Neocrex erythrops (Sclater, 1867) & Gallineta de Pico Rojo & Paint-billed Crake & H & & \\
\hline RALLIDAE & Pardirallus maculatus (Boddaert, 1783) & Rascón Moteado & Spotted Rail & $\mathbf{H}$ & & \\
\hline RALLIDAE & Pardirallus sanguinolentus (Swainson, 1838) & Rascón Plomizo & Plumbeous Rail & $\mathbf{H}$ & & \\
\hline RALLIDAE & Gallinula chloropus (Linnaeus, 1758) & Polla de Agua Común & Common Gallinule & $H, A$ & & \\
\hline RALLIDAE & Porphyrio martinica (Linnaeus, 1766) & Polla de Agua Morada (Polla Sultana) & Purple Gallinule & $\mathbf{H}$ & & \\
\hline RALLIDAE & Fulica ardesiaca (Tschudi, 1843) & Gallareta Andina & Slate-colored Coot & $\mathbf{H}$ & & \\
\hline CHARADRIIDAE & Pluvialis dominica (Müller, 1776) & Chorlo Dorado Americano & American Golden-Plover & $\mathrm{H}, \mathrm{M}$ & & \\
\hline CHARADRIIDAE & Pluvialis squatarola (Linnaeus, 1758) & Chorlo Gris & Black-bellied Plover & $\mathrm{H}, \mathrm{M}$ & & \\
\hline CHARADRIIDAE & Charadrius semipalmatus (Bonaparte, 1825) & Chorlo Semipalmado & Semipalmated Plover & $\mathrm{H}, \mathrm{M}$ & & \\
\hline CHARADRIIDAE & Charadrius vociferus (Linnaeus, 1758) & Chorlo Gritón (Tic-til) & Killdeer & $\mathrm{H}, \mathrm{A}$ & & \\
\hline CHARADRIIDAE & Charadrius alexandrinus (Linnaeus, 1758) & Chorlo Nevado & Snowy Plover & $\mathrm{H}, \mathrm{M}$ & & \\
\hline CHARADRIIDAE & Charadrius collaris (Vieillot, 1818) & Chorlo Acollarado & Collared Plover & $\mathrm{H}, \mathrm{M}$ & & \\
\hline CHARADRIIDAE & Oreopholus ruficollis (Wagler, 1829) & Chorlo de Campo & Tawny-throated Dotterel & A & & \\
\hline HAEMATOPODIDAE & Haematopus palliatus (Temminck, 1820) & Ostrero Americano & American Oystercatcher & $\mathbf{H}, \mathbf{M}$ & & \\
\hline RECURVIROSTRIDAE & Himantopus mexicanus (Müller, 1776) & Cigüeñuela de Cuello Negro (Perrito) & Black-necked Stilt & $H, A, M$ & & \\
\hline BURHINIDAE & Burhinus superciliaris (Tschudi, 1843) & Alcaraván Huerequeque & Peruvian Thick-knee & H, A & & \\
\hline
\end{tabular}


Tabla 1. Aves de los Humedales de Puerto Eten, Lambayeque, Perú.

Hábitat. H: Humedal, M: Mar y playa, A: Arbustos, árboles y agricultura Endemismo. T: EBA Tumbesina, P: Perú Amenaza. EN: En Peligro, VU: Vulnerable, LC: Preocupación menor

\begin{tabular}{|c|c|c|c|c|c|c|}
\hline Familia & Nombre Científico & Nombre en Español & Nombre en Ingles & 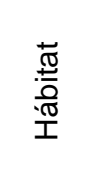 & 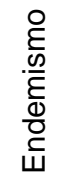 & 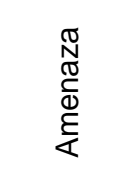 \\
\hline SCOLOPACIDAE & Limnodromus griseus (Gmelin, 1789) & Agujeta de Pico Corto & Short-billed Dowitcher & $H, M$ & & \\
\hline SCOLOPACIDAE & Limosa haemastica (Linnaeus, 1758) & Aguja de Mar & Hudsonian Godwit & $\mathrm{H}, \mathbf{M}$ & & \\
\hline SCOLOPACIDAE & Numenius phaeopus (Linnaeus, 1758) & Zarapito Trinador & Whimbrel & $\mathrm{H}, \mathbf{M}$ & & \\
\hline SCOLOPACIDAE & Actitis macularius (Linnaeus, 1766) & Playero Coleador & Spotted Sandpiper & $\mathrm{H}, \mathbf{M}$ & & \\
\hline SCOLOPACIDAE & Tringa melanoleuca (Gmelin, 1789) & Playero Pata Amarilla Mayor & Greater Yellowlegs & $\mathrm{H}, \mathrm{M}$ & & \\
\hline SCOLOPACIDAE & Tringa flavipes (Gmelin, 1789) & Playero Pata Amarilla Menor & Lesser Yellowlegs & $\mathrm{H}, \mathbf{M}$ & & \\
\hline SCOLOPACIDAE & Tringa semipalmata (Gmelin, 1789) & Playero de Ala Blanca & Willet & $H, M$ & & \\
\hline SCOLOPACIDAE & Arenaria interpres (Linnaeus, 1758) & Vuelvepiedras Rojizo & Ruddy Turnstone & $\mathrm{H}, \mathbf{M}$ & & \\
\hline SCOLOPACIDAE & Aphriza virgata (Gmelin, 1789) & Chorlo de las Rompientes & Surfbird & $H, M$ & & \\
\hline SCOLOPACIDAE & Calidris canutus (Linnaeus, 1758) & Playero de Pecho Rufo & Red Knot & $\mathrm{H}, \mathrm{M}$ & & \\
\hline SCOLOPACIDAE & Calidris alba (Pallas, 1764) & Playero Arenero & Sanderling & $\mathrm{H}, \mathrm{M}$ & & \\
\hline SCOLOPACIDAE & Calidris pusilla (Linnaeus, 1766) & Playerito Semipalmado & Semipalmated Sandpiper & $\mathrm{H}, \mathbf{M}$ & & \\
\hline SCOLOPACIDAE & Calidris mauri (Cabanis, 1857) & Playerito Occidental & Western Sandpiper & $\mathrm{H}, \mathbf{M}$ & & \\
\hline SCOLOPACIDAE & Calidris minutilla (Vieillot, 1819) & Playerito Menudo & Least Sandpiper & $\mathrm{H}, \mathbf{M}$ & & \\
\hline SCOLOPACIDAE & Calidris fuscicollis (Vieillot, 1819) & Playerito de Lomo Blanco & White-rumped Sandpiper & $\mathbf{H}$ & & \\
\hline SCOLOPACIDAE & Calidris bairdii (Coues, 1861) & Playerito de Baird & Baird's Sandpiper & $\mathbf{H}, \mathbf{M}$ & & \\
\hline SCOLOPACIDAE & Calidris melanotos (Vieillot, 1819) & Playero Pectoral & Pectoral Sandpiper & $\mathrm{H}, \mathbf{M}$ & & \\
\hline SCOLOPACIDAE & Calidris himantopus (Bonaparte, 1826) & Playero de Patas Largas & Stilt Sandpiper & $H, M$ & & \\
\hline SCOLOPACIDAE & Phalaropus tricolor (Vieillot, 1819) & Faláropo Tricolor & Wilson's Phalarope & $\mathrm{H}, \mathrm{M}$ & & \\
\hline SCOLOPACIDAE & Phalaropus lobatus (Linnaeus, 1758) & Faláropo de Pico Fino & Red-necked Phalarope & $\mathrm{H}, \mathbf{M}$ & & \\
\hline SCOLOPACIDAE & Phalaropus fulicarius (Linnaeus, 1758) & Faláropo de Pico Grueso & Red Phalarope & $H, M$ & & \\
\hline THINOCORIDAE & Thinocorus rumicivorus (Eschscholtz, 1829) & Agachona Chica & Least Seedsnipe & A & & \\
\hline LARIDAE & Chroicocephalus cirrocephalus (Vieillot, 1818) & Gaviota de Capucho Gris & Gray-hooded Gull & $\mathbf{H}, \mathbf{M}$ & & \\
\hline LARIDAE & Leucophaeus modestus (Tschudi, 1843) & Gaviota Gris & Gray Gull & $\mathrm{H}, \mathbf{M}$ & & \\
\hline LARIDAE & Leucophaeus atricilla (Linnaeus, 1758) & Gaviota Reidora & Laughing Gull & $H, M$ & & \\
\hline LARIDAE & Leucophaeus pipixcan (Wagler, 1813) & Gaviota de Franklin & Franklin's Gull & $\mathrm{H}, \mathbf{M}$ & & \\
\hline LARIDAE & Larus belcheri (Vigors, 1829) & Gaviota Peruana & Band-tailed Gull & $H, M$ & & \\
\hline LARIDAE & Larus dominicanus (Lichtenstein, 1823) & Gaviota Dominicana & Kelp Gull & $\mathrm{H}, \mathbf{M}$ & & \\
\hline LARIDAE & Sternula Iorata (Philippi \& Landbeck, 1861) & Gaviotín Peruano & Peruvian Tern & $\mathrm{H}, \mathrm{M}$ & & $\mathrm{EN} / \mathrm{VU}$ \\
\hline
\end{tabular}


Tabla 1. Aves de los Humedales de Puerto Eten, Lambayeque, Perú.

Hábitat. H: Humedal, M: Mar y playa, A: Arbustos, árboles y agricultura Amenaza. EN: En Peligro, vU: Vulnerable, LC: Preocupación menor

\begin{tabular}{|c|c|c|c|c|c|c|}
\hline Familia & Nombre Científico & Nombre en Español & Nombre en Ingles & $\begin{array}{l}\frac{\pi}{\pi} \\
\stackrel{\pi}{\frac{\pi}{\pi}} \\
\frac{\pi}{I}\end{array}$ & 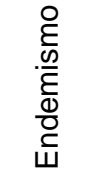 & 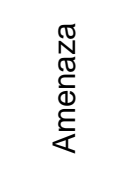 \\
\hline LARIDAE & Gelochelidon nilotica (Gmelin, 1789) & Gaviotín de Pico Negro & Gull-billed Tern & M & & \\
\hline LARIDAE & Larosterna inca (Lesson, 1827) & Gaviotín Zarcillo & Inca Tern & M & & NT / VU \\
\hline LARIDAE & Sterna hirundo (Linnaeus, 1758) & Gaviotín Común & Common Tern & M & & \\
\hline LARIDAE & Sterna paradisaea (Pontoppidan, 1763) & Gaviotín del Ártico & Arctic Tern & M & & \\
\hline LARIDAE & Sterna hirundinacea (Lesson, 1831) & Gaviotín Sudamericano & South American Tern & M & & LC I VU \\
\hline LARIDAE & Thalasseus elegans (Gambel, 1849) & Gaviotín Elegante & Elegant Tern & $\mathbf{H}, \mathbf{M}$ & & \\
\hline LARIDAE & Thalasseus sandvicensis (Latham, 1787) & Gaviotín de Patas Negras & Sandwich Tern & $\mathbf{M}$ & & \\
\hline LARIDAE & Thalasseus maximus (Boddaert, 1783) & Gaviotín Real & Royal Tern & $\mathbf{H}, \mathbf{M}$ & & \\
\hline RYNCHOPIDAE & Rynchops niger (Linnaeus, 1758) & Rayador Negro & Black Skimmer & $\mathbf{H}, \mathbf{M}$ & & \\
\hline COLUMBIDAE & Columbina minuta (Linnaeus, 1766) & Tortolita Menuda & Plain-breasted Ground-Dove & A & & \\
\hline COLUMBIDAE & Columbina cruziana (Prévost, 1842) & Tortolita Peruana & Croaking Ground-Dove & $\mathbf{H}, \mathbf{A}$ & & \\
\hline COLUMBIDAE & Zenaida meloda (Tschudi, 1843) & Tórtola Melódica (Cuculí) & West Peruvian Dove & A & & \\
\hline COLUMBIDAE & Zenaida auriculata (Des Murs, 1847) & Tórtola Orejuda & Eared Dove & A & & \\
\hline PSITTACIDAE & Forpus coelestis (Lesson, 1847) & Perico Esmeralda (Esmeraldita) & Pacific Parrotlet & $\mathbf{H}, \mathbf{A}$ & $\mathbf{T}$ & \\
\hline CUCULIDAE & Crotophaga sulcirostris (Swainson, 1827) & Garrapatero de Pico Estriado (Chiclón) & Groove-billed Ani & $\mathbf{H}, \mathbf{A}$ & & \\
\hline STRIGIDAE & Glaucidium peruanum (König, 1991) & Lechucita Peruana & Peruvian Pigmy-Owl & A & & \\
\hline STRIGIDAE & Athene cunicularia (Molina, 1782) & Lechuza Terrestre & Burrowing Owl & $\mathrm{H}, \mathrm{A}$ & & \\
\hline STRIGIDAE & Asio flammeus (Pontoppidan, 1763) & Lechuza de Oreja Corta & Short-eared Owl & $\mathbf{H}, \mathbf{A}$ & & \\
\hline CAPRIMULGIDAE & Chordeiles acutipennis (Hermann, 1783) & Chotacabras Menor & Lesser Nighthawk & $\mathrm{H}, \mathrm{A}$ & & \\
\hline TROCHILIDAE & Myrtis fanny (Lesson, 1838) & Estrellita Collaripurpura & Purple-collared Woodstar & A & & \\
\hline TROCHILIDAE & Rhodopis vesper (Lesson, 1829) & Colibrí de Oasis & Oasis Hummingbird & A & & \\
\hline TROCHILIDAE & Thaumastura cora (Lesson, 1827) & Colibrí de Cora & Peruvian Sheartail & A & & \\
\hline TROCHILIDAE & Myrmia micrura (Gould, 1854) & Estrellita Colicorta & Short-tailed Woodstar & A & $\mathbf{T}$ & \\
\hline TROCHILIDAE & Amazilia amazilia Gould, 1860) & Colibrí de Vientre Rufo & Amazilia Hummingbird & A & & \\
\hline ALCEDINIDAE & Megaceryle torquata (Linnaeus, 1766) & Martín Pescador Grande & Ringed Kingfisher & $\mathbf{H}$ & & \\
\hline ALCEDINIDAE & Chloroceryle americana (Gmelin, 1788) & Martín Pescador Verde & Green Kingfisher & $\mathbf{H}$ & & \\
\hline FURNARIIDAE & Geositta peruviana (Lafresnaye, 1847) & Minero Peruano (Pampero) & Coastal Miner & $\mathrm{H}, \mathrm{A}$ & $\mathbf{T} / \mathbf{P}$ & \\
\hline FURNARIIDAE & Furnarius leucopus (Swainson, 1837) & Hornero Pacifico (Chilala) & Pale-legged Hornero & A & & \\
\hline FURNARIIDAE & Phleocryptes melanops (Vieillot, 1817) & Totorero & Wren-like Rushbird & $\mathbf{H}$ & & \\
\hline
\end{tabular}


Tabla 1. Aves de los Humedales de Puerto Eten, Lambayeque, Perú.

\begin{tabular}{|c|c|c|c|c|c|c|}
\hline & \multicolumn{4}{|c|}{$\begin{array}{r}\text { Hábitat. H: Humedal, M: Mar y playa, A: Arbustos, árboles y agricultura } \\
\text { Endemismo. T: EBA Tumbesina, P: Perú } \\
\text { Amenaza. EN: En Peligro, VU: Vulnerable, LC: Preocupación menor }\end{array}$} \\
\hline Familia & Nombre Científico & Nombre en Español & Nombre en Ingles & 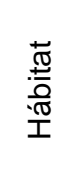 & 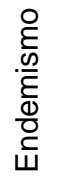 & 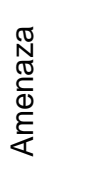 \\
\hline TYRANNIDAE & Camptostoma obsoletum (Temminck, 1824) & Mosquerito Silbador & Southern Beardless-Tyrannulet & A & & \\
\hline TYRANNIDAE & Euscarthmus meloryphus (Wied, 1831) & Tirano-Pigmeo de Corona Leonada & Tawny-crowned Pygmy-Tyrant & A & & \\
\hline TYRANNIDAE & Tachuris rubrigastra (Vieillot, 1817) & Siete Colores de la Totora & Many-colored Rush-Tyrant & $\mathbf{H}$ & & \\
\hline TYRANNIDAE & Todirostrum cinereum (Linnaeus, 1766) & Espatulilla Comun & Common Tody-Flycatcher & A & & \\
\hline TYRANNIDAE & Myiophobus fasciatus (Müller, 1776) & Mosquerito de Pecho Rayado & Bran-colored Flycatcher & A & & \\
\hline TYRANNIDAE & Pyrocephalus rubinus (Boddaert, 1783) & Mosquero Bermellón (Putilla) & Vermilion Flycatcher & $\mathrm{H}, \mathrm{A}$ & & \\
\hline TYRANNIDAE & Muscigralla brevicauda(d'Orbigny \& Lafresnaye, 1837) & Dormilona de Cola Corta & Short-tailed Field-Tyrant & A & & \\
\hline TYRANNIDAE & Myiodynastes bairdii (Gambel, 1847) & Mosquero de Baird (Celosa) & Baird's Flycatcher & A & $\mathbf{T}$ & \\
\hline TYRANNIDAE & Tyrannus melancholicus (Vieillot, 1819) & Tirano Tropical & Tropical Kingbird & A & & \\
\hline VIREONIDAE & Cyclarhis gujanensis (Gmelin, 1789) & Vireón Cejirrufa & Rufous-browed Peppershrike & A & & \\
\hline HIRUNDINIDAE & Pygochelidon cyanoleuca (Vieillot, 1817) & Golondrina Azul y Blanco (Santa Rosita) & Blue-and-white Swallow & $\mathrm{H}, \mathrm{A}$ & & \\
\hline HIRUNDINIDAE & Stelgidopteryx ruficollis (Vieillot, 1817) & Golondrina Alirrasposa Sureña & Southern Rough-winged Swallow & $\mathrm{H}, \mathrm{A}$ & & \\
\hline HIRUNDINIDAE & Progne chalybea (Gmelin, 1789) & Martín Pechigris & Gray-breasted Martin & $\mathrm{H}, \mathrm{A}$ & & \\
\hline HIRUNDINIDAE & Riparia riparia (Linnaeus, 1758) & Golondrina Ribereña & Bank Swallow & $\mathrm{H}, \mathrm{A}$ & & \\
\hline HIRUNDINIDAE & Hirundo rustica (Linnaeus, 1758) & Golondrina Tijereta & Barn Swallow & $\mathrm{H}, \mathrm{A}$ & & \\
\hline HIRUNDINIDAE & Petrochelidon rufocollaris (Peale, 1848) & Golondrina de Collar Castaño & Chestnut-collared Swallow & A & & \\
\hline TROGLODYTIDAE & Troglodytes aedon (Vieillot, 1809) & Cucarachero Común & House Wren & A & & \\
\hline TROGLODYTIDAE & Campylorhynchus fasciatus (Swainson, 1837) & Cucarachero Ondeado (Choqueco, Coquila) & Fasciated Wren & A & & \\
\hline TROGLODYTIDAE & Thryothorus superciliaris (Lawrence, 1869) & Cucarachero Cejón (Ruiseñor) & Superciliated Wren & A & $\mathbf{T}$ & \\
\hline POLIOPTILIDAE & Polioptila plumbea (Gmelin, 1788) & Perlita Tropical & Tropical Gnatcatcher & A & & \\
\hline MIMIDAE & Mimus longicaudatus (Tschudi, 1844) & Calandria de Cola Larga (Chisco) & Long-tailed Mockingbird & A & & \\
\hline MOTACILLIDAE & Anthus lutescens (Pucheran, 1855) & Cachirla Amarillenta & Yellowish Pipit & $\mathrm{H}, \mathrm{A}$ & & \\
\hline THRAUPIDAE & Thraupis episcopus (Linnaeus, 1766) & Tangara Azuleja (Luisa) & Blue-gray Tanager & A & & \\
\hline THRAUPIDAE & Conirostrum cinereum (Lafresnaye \& d'Orbigny, 1838) & Pico de Cono Cinéreo & Conirostrum cinereum & A & & \\
\hline INCERTAE SEDIS & Coereba flaveola (Linnaeus, 1758) & Reinita Mielera & Bananaquit & A & & \\
\hline EMBERIZIDAE & Zonotrichia capensis (Müller, 1776) & Gorrión de Collar Rufo & Rufous-collared Sparrow & $\mathrm{H}, \mathrm{A}$ & & \\
\hline EMBERIZIDAE & Poospiza hispaniolensis (Bonaparte, 1851l) & Monterita Acollarada & Collared Warbling-Finch & A & & \\
\hline EMBERIZIDAE & Sicalis flaveola (Linnaeus, 1766) & Chirigüe Azafranado (Botón de Oro) & Saffron Finch & A & & \\
\hline EMBERIZIDAE & Volatinia jacarina (Linnaeus, 1766) & Semillerito Negro Azulado (Saltapalito) & Blue-black Grassquit & A & & \\
\hline
\end{tabular}


Tabla 1. Aves de los Humedales de Puerto Eten, Lambayeque, Perú.

\begin{tabular}{|c|c|c|c|c|c|c|}
\hline & & & \multicolumn{4}{|c|}{$\begin{array}{r}\text { Hábitat. H: Humedal, M: Mar y playa, A: Arbustos, árboles y agricultura } \\
\text { Endemismo. T: EBA Tumbesina, P: Perú } \\
\text { Amenaza. EN: En Peligro, VU: Vulnerable, LC: Preocupación menor }\end{array}$} \\
\hline Familia & Nombre Científico & Nombre en Español & Nombre en Ingles & $\begin{array}{l}\frac{\pi}{0} \\
\frac{\pi}{\pi} \\
\frac{\pi}{1}\end{array}$ & 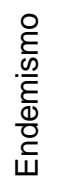 & 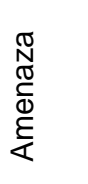 \\
\hline EMBERIZIDAE & Sporophila corvina (P.L. Sclater, 1859) & Espiguero Variable & Variable Seedeater & A & & \\
\hline EMBERIZIDAE & Sporophila peruviana (Lesson, 1842) & Espiguero Pico de Loro (Choclopococho) & Parrot-billed Seedeater & A & & \\
\hline EMBERIZIDAE & Sporophila telasco (Taczanowski, 1874) & Espiguero de Garganta Castaña & Chestnut-throated Seedeater & A & & \\
\hline CARDINALIDAE & Pheucticus chrysogaster (Lesson, 1832) & Picogrueso Ventridorado (Lingan) & Golden-bellied Grosbeak & A & & \\
\hline PARULIDAE & Geothlypis aequinoctialis (Gmelin, 1789) & Reinita Equinoccial & Masked Yellowthroat & A & & \\
\hline ICTERIDAE & Dives warszewiczi (Cabanis, 1861) & Tordo de Matorral (Chihuin) & Scrub Blackbird & A & & \\
\hline ICTERIDAE & Molothrus bonariensis (Gmelin, 1789) & Tordo Brilloso & Shiny Cowbird & A & & \\
\hline ICTERIDAE & Sturnella bellicosa(de Filippi, 1847) & Pecho Colorado Peruano (Peche) & Peruvian Meadowlark & $\mathrm{H}, \mathrm{A}$ & & \\
\hline FRINGILLIDAE & Carduelis magellanica (Vieillot, 1805) & Jilguero Encapuchado & Hooded Siskin & A & & \\
\hline PASSERIDAE & Passer domesticus (Linnaeus, 1758) & Gorrión Casero & House Sparrow & A & & \\
\hline
\end{tabular}

${ }^{1}$ BirdLife International. fernando.angulo@birdlife.org.
${ }^{2}$ Cornell Lab of Ornithology, 159 Sapsucker Woods Road, Ithaca NY 14850, United States. tss62@cornell.edu.
${ }^{3}$ Universidad Nacional Pedro Ruiz Gallo, Los Laureles 621. UPIS - José Leonardo Ortiz, Chiclayo, Perú. elberthedevaly@gmail.com. 